\title{
Christopher Hammond: The basics of crystallography and diffraction. Third edition
}

\author{
Oxford University Press for the International Union of Crystallography, \\ Oxford, UK, 2009
}

István Hargittai

Published online: 19 June 2009

(C) Springer Science+Business Media, LLC 2009

This is one of the latest additions to the International Union of Crystallography (IUCr) Texts on Crystallography, which is one of the two book series the Union runs. The other is the IUCr Monographs on Crystallography. These projects were started in the early 1990s and have proved very useful for the world crystallographic community and beyond.

The fact that Hammond's monograph has reached its third edition speaks of its success, which is equally applicable to its scientific content and its pedagogy. As the title indicates, both crystals and crystal structure research and the diffraction techniques are given ample exposure in the monograph.

The first six chapters deal with the aspects of crystallography; crystals and their structures, two-dimensional patterns and lattices, Bravais lattices and crystals systems, symmetries, both point groups and space groups, lattice planes, Miller indices, and finally, the reciprocal lattice. This is augmented by a later chapter on stereographic projection. Six chapters, the second half of the book, are devoted to diffraction, covering light, X-rays, and electrons, concluding with a brief description of Fourier analysis in diffraction and image formation.

I would like to single out three features of this book. One is the attention to history, which not only makes the presentation livelier, but is also very informative, and biographical notes are given in an Appendix. The other is that while X-ray diffraction is given the most detailed presentation, electron diffraction is not neglected. The third is that the latest development in what is often called generalized crystallography-quasicrystals, etc.-is not merely mentioned in passing or added for completeness. Rather, it is organically built into the rest of the material.

The utility of this volume for teaching is greatly enhanced by exercises at the ends of the chapters and by the answers to the exercises at the end of the volume. There is also an annotated reading list. As this is already the third edition, it did not seem warranted to present a detailed review. Those familiar with the previous editions will notice, as one of the expansions, the discussion of layer group symmetriesindeed a useful addition. The number of crystal structures presented as examples has also been increased. There are other extensions, including an entirely new chapter, referred to above, on Fourier analysis and image formation.

The book is nicely illustrated and attractively produced. It is warmly recommended to all students and researchers in crystallography, including chemists, condensed-state physicists, materials scientists, and others who are interested in the structures of crystals and in how they are determined.

\footnotetext{
I. Hargittai $(\bowtie)$

Department of Inorganic and Analytical Chemistry and Materials Structure and Modeling Research Group of the Hungarian Academy of Sciences, Budapest University of Technology and Economics, P.O. Box 91, 1521 Budapest, Hungary

e-mail: istvan.hargittai@gmail.com
} 